\title{
A rare case of a newborn with urinary tract infection by Raoutella ornithinolytica
}

\author{
Esra Akyuz Ozkan ${ }^{1, ~}$, Hashem E. Khosroshahi ${ }^{2}$, Neziha Yilmaz ${ }^{3}$, U. Aliye Gecit ${ }^{1}$, Esra Domur $^{1}$ \\ ${ }^{1}$ Department of Pediatrics, Bozok University Faculty of Medicine, Yozgat, Turkey \\ ${ }^{2}$ Department of Pediatric Cardiology, Bozok University Faculty of Medicine, Yozgat, Turkey \\ ${ }^{3}$ Department of Microbiology, Bozok University Faculty of Medicine, Yozgat, Turkey
}

Email address:

esra.akyuz@mynet.com (E. A. Ozkan)

To cite this article:

Esra Akyuz Ozkan, Hashem E. Khosroshahi, Neziha Yilmaz, U. Aliye Gecit, Esra Domur. A Rare Case of a Newborn with Urinary Tract Infection by Raoutella Ornithinolytica. American Journal of Health Research. Vol. 2, No. 6, 2014, pp. 375-377.

doi: 10.11648/j.ajhr.20140206.19

\begin{abstract}
Raoultella ornithinolytica is a gram negative aerobic bacillus belonging in the family Enterobacteriaceae that is a known but extremely rare pathogen which causes infections in humans. We report on a newborn with urinary tract infection caused by Raoultella ornithinolytica. The present case is the first newborn patient with urinary tract infection and only the third newborn found infected with Raoultella ornithinolytica reported in the literature.
\end{abstract}

Keywords: Newborn, Raoultella Ornithinolytica, Urinary Tract Infection

\section{Introduction}

Raoultella ornithinolytica (R. ornithinolytica) is a gramnegative aerobic bacillus of the family Enterobacteriaceae. It is found in aquatic environments, fish and insects and causes invasive infections in humans [1]. R. ornithinolytica has also been isolated from infected root canal dentin because of alkali resistance [2]. R. ornithinolytica produces histamine and causes fish poisoning [3] which presents clinically with red skin flushing, headache, abdominal cramps and in rare cases, tachycardia, hypotension and bronchospasm [4].

Here, we report on a newborn presenting with urinary tract infection caused by R. ornithinolytica. To the best of our knowledge this is only the third newborn case of human infection caused by R. ornithinolytica that has been reported in the literature.

\section{Case Report}

A 21 day-old boy was referred to our hospital with primary complaints of restlessness and vomiting for about two weeks. He was born to a 27-year-old mother, at term with caesarean section. Birth weight was $3200 \mathrm{~g}$; height was $50 \mathrm{~cm}$ and observed without any pre- or perinatal complication. On postnatal examination he had pes eqinovarus and 1/6 systolic murmur at left sternal border. He had no palpable mass or organomegaly and his external genitalia were normal. On second examination when referred to us, his body temperature was $36.2^{\circ} \mathrm{C}$ and heart rate was 110 per minute; weight $4200 \mathrm{~g}$; and height $52 \mathrm{~cm}$. Respiratory, abdominal and neurological examinations were all within normal limits. $\mathrm{He}$ had no flushing and no rash. Routine laboratory showed a white blood cell count of 7.52 cells $/ \mathrm{mm}^{3}$, erythrocyte sedimentation rate of $15 \mathrm{~mm} / \mathrm{h}$, and C-reactive protein level of $0.1 \mathrm{mg} / \mathrm{L}$. Serum urea, creatinine, electrolytes, calcium, phosphate, uric acid and liver functions were within normal limits. Abdominal ultrasound was performed in terms of the urinary system malformations and the results were normal. To investigate the $1 / 6$ systolic murmur he underwent echocardiography and a sinus venosus type atrial septal defect was determined. He had pes eqinovarus and he was cast by orthopedics.

Because of the patient's restlessness and vomiting, urine culture was taken and it was positive for a gram-negative bacillus identified as R. ornithinolytica ( $>10^{5}$ colonies) [Fig 1]. The urine was cultivated on EMB and blood agar with 10 $\mu \mathrm{L}$ standard loop. The colonies proliferated in both agars selected for gram staining and gram negative bacilli were isolated. Bacterial identification was carried out using the VITEK-2 compact system. An antibiogram for $\mathrm{R}$. 
ornithinolytica demonstrated susceptibility to aminoglycosides, cefepime, carbapenems and trimethoprimsulfamethoxazole and resistant to ampicillin and third

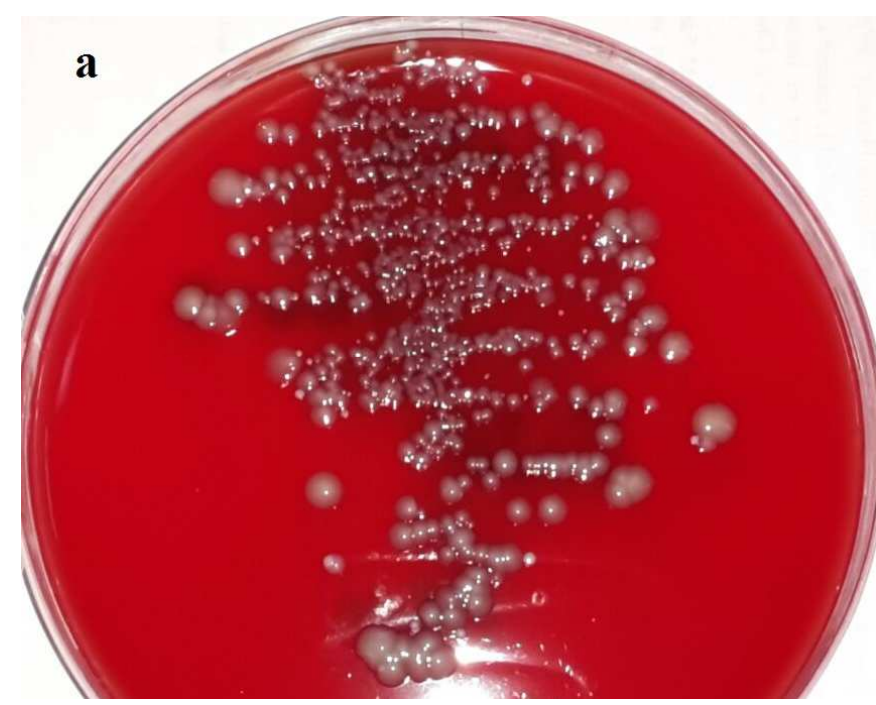

generation cephalosporin. Amikacine sulfate was administered $15 \mathrm{mg} / \mathrm{kg} / \mathrm{day} / \mathrm{qd}$. Symptoms subsided after receiving treatment. Follow-up cultures were sterile.

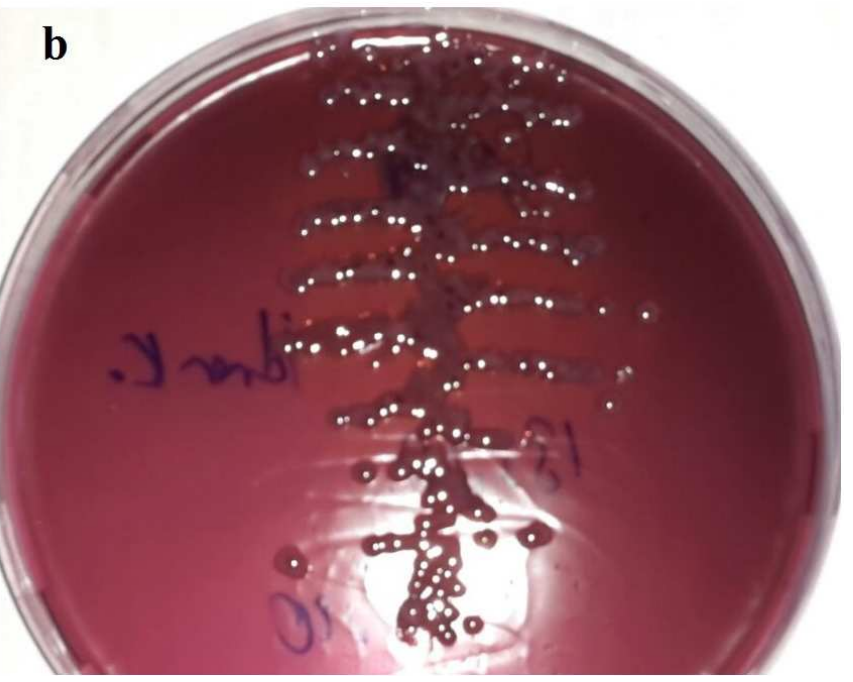

Fig 1. a-blood agar, b-EMB agar. Colonies of gram negative bacteria were isolated. Bacteriel identification was performed using the VITEK-2 compact system.

\section{Discussion}

R. ornithinolytica was first described by Kosako et al in 1989 [5]. Monnet and Freney used growth tests to differentiate Klebsiella ornithinolytica and Klebsiella planticola and they isolated another species capable of producing histamine 1994 [6].

So far there are only two other newborn cases with R. ornithinolytica infections reported in the literature. The first case was a preterm baby presenting with septic shock and purpura fulminans and died at 19 days of age [7]. Based on this case, it has been suggested that R. ornithinolytica may cause fatal neonatal infection, especially in preterm infants [7]. The second case of R. ornithinolytica presented with bacteremia, asplenia, congenital cardiac defects and visceral heterotaxy [8]. Our case is the third newborn found to be infected with R. ornithinolytica bacterium. In the present case, there was no fish poisoning symptoms, such as red skin flushing, tachycardia, hypotension and bronchospasm. After 10 days treatment his symptoms were resolved, control urine culture was negative and he was discharged.

Among total 10 adult patients with R. ornithinolytica bacteremia reported in the literature, the majority of cases suffered from biliary infection. In the latter cases the pathogens were susceptible to both third-generation cephalosporin and fluoroquinolone and all the patients were treated effectively with antibiotics. These cases showed that elderly patients with biliary interventions, biliary tract diseases, or malignancy bear high risk for R. ornithinolytica bacteremia [9].

$\mathrm{R}$. ornithinolytica is an encapsulated bacterium and patients with hyposplenism/asplenia are considered high risk group affected by this pathogen. There is only one infant who has been reported with congenital asplenia infected with $\mathrm{R}$. ornithinolytica [8]. In our case asplenia was ruled out by abdominal ultrasound. As in our patient, R. ornithinolytica was reported to be resistant to ampicillin [10] and this resistance can be associated with the presence of $\beta$ lactamases [11]. In this report, R. ornithinolytica was also resistant to third generation cephalosporins and susceptible to amoxicillin clavulanate, piperacillin tazobactam, carbapenem, aminoglycosides and trimethoprim-sulfamethoxazole.

Patients who were infected with $\mathrm{R}$. ornithinolytica, are considered to have a poor prognosis due to its belonging to the genus Klebsiella, which is highly virulent in humans. However, the outcomes of the treatment for R. ornithinolytica bacteremia were satisfactory for almost all cases.

In conclusion, this is the first case report that illustrates isolation of the extremely rare pathogen R. ornithinolytica from the urinary tract of a newborn. He demonstrated an excellent prognosis with antibiotic therapy as reported in the literature.

\section{References}

[1] Morais VP, Daporta MT, Bao AF, Campello MG, Andrés GQ. Enteric fever-like syndrome caused by Raoultella ornithinolytica (Klebsiella ornithinolytica). J Clin Microbiol 2009; 47(3):868-9.

[2] Nakajo K, Nakazawa F, Iwaku M, Hoshino E. Alkali-resistant bacteria in root canal systems. Oral Microbiol Immunol 2004;19(6):390-4.

[3] Kanki M, Yoda T, Tsukamoto T, Shibata T. Klebsiella pneumoniae produces no histamine: Raoultella planticola and Raoultella ornithinolytica strains are histamine producers. Appl Environ Microbiol 2002; 68(7):3462-6. 
[4] Ferran M, Yébenes M. Flushing associated with scombroid fish poisoning. Dermatol Online J 2006; 12(6):15.

[5] Kosako Y, Tamura K, Sakazaki R, Miki K. Klebsiella ornithinolytica sp. nov., formerly known as ornithine-positive Klebsiella oxytoca. Curr Microbiol 1989;18(4):201-6

[6] Monnet D, Freney J. Method for differentiating Klebsiella planticola and Klebsiella terrigena from other Klebsiella species. J Clin Microbiol 1994;32(4):1121-2.

[7] G Sandal, M Ozen. Fatal Raoultella ornithinolytica sepsis and purpura fulminans in a preterm newborn. Indian Journal of Paediatric Dermatology 2014;15(1):24

[8] Mau N, Ross LA. Raoultella ornithinolytica bacteremia in an infant with visceral heterotaxy. Pediatr Infect Dis J 2010; 29(5):477-8.
[9] Haruki Y, Hagiya H, Sakuma A, Murase T, Sugiyama T, Kondo S. Clinical characteristics of Raoultella ornithinolytica bacteremia: a case series and literature review. J Infect Chemother 2014; 20(9):589-91.

[10] Hostacká A, Klokocníková. Antibiotic susceptibility, serum response and surface properties of Klebsiella species. Microbios 2001;104(408):115-24.

[11] Walckenaer E, Poirel L, Leflon-Guibout V, Nordmann P, Nicolas-Chanoine MH. Genetic and biochemical characterization of the chromosomal class A beta-lactamases of Raoultella (formerly Klebsiella) planticola and Raoultella ornithinolytica. Antimicrob Agents Chemother 2004;48(1):305-12. 